\title{
Mass and width of the Delta resonance using complex-mass renormalization
}

\author{
Y. Ünal ${ }^{1,2, \star}$, T. Bauer ${ }^{1}$, A. Küçükarslan ${ }^{2}$, and S. Scherer ${ }^{1}$ \\ ${ }^{1}$ PRISMA Cluster of Excellence, Institut für Kernphysik, Johannes Gutenberg-Universität Mainz, D-55099 \\ Mainz, Germany \\ ${ }^{2}$ Physics Department, Çanakkale Onsekiz Mart University, 17100 Çanakkale, Turkey
}

\begin{abstract}
The pole mass and width of the Delta resonance are calculated in the relativistic chiral effective field theory approach. We choose a systematic power-counting procedure by applying the complex-mass scheme (CMS).
\end{abstract}

\section{Introduction}

At low energies, the chiral perturbation theory (ChPT) [1-3] provides a successful description of the strong interaction of pion and nucleon-based effective field theory (EFT) of quantum choromodynamics (QCD). Inclusion of heavy and especially resonant degrees of freedom in EFT becomes more complicated to set up a systematic counting scheme [4]. Power counting violating imaginary terms cannot be absorbed by the use of traditional renormalization schemes. Therefore, we can apply CMS to restore a systematic counting scheme [5].

In the current study, we determine the mass and width of the delta resonance in relativistic chiral EFT up to order $O\left(q^{3}\right)$. We write the most general effective Lagrangians as $[6,7]$

$$
\begin{gathered}
\mathcal{L}=\mathcal{L}_{0}+\mathcal{L}_{\pi \Delta}+\mathcal{L}_{\pi N \Delta}+\mathcal{L}_{\Delta}^{(2)}, \\
\mathcal{L}_{0}=\bar{\Psi}\left(i \not D-m_{N 0}\right) \Psi-\bar{\psi}_{\mu} P^{\frac{3}{2}}\left[\left(i \not D-m_{\Delta 0}\right) g^{\mu \nu}\right. \\
\left.-i\left(\gamma^{\mu} D^{v}+\gamma^{v} D^{\mu}\right)+i \gamma^{\mu} \not D \gamma^{v}+m_{\Delta 0} \gamma^{\mu} \gamma^{v}\right] P^{\frac{3}{2}} \psi_{v},
\end{gathered}
$$

where $\mathrm{N}$ denotes nucleon isospin doublet with bare mass $m_{N 0} . \psi_{v}$ are the vector-spinor isovectorisospinor Rarita Schwinger fields of the $\Delta$ resonance [8] with bare mass $m_{\Delta 0}, P^{\frac{3}{2}}$ is the isospin-3/2 projector [9].

\footnotetext{
^e-mail: yaseminunal@comu.edu.tr
} 


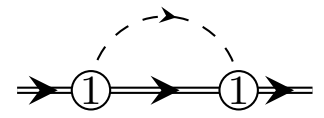

(a)

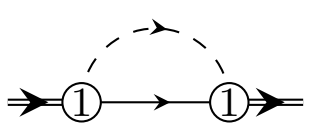

(b)

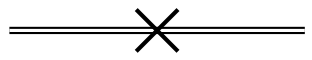

(c)

Figure 1. One-loop self energy diagrams of the delta. The dashed, straight, and double lines correspond to pion, nucleon and delta, respectively.

$$
\begin{aligned}
\mathcal{L}_{\pi \Delta}^{(1)}= & -\bar{\psi}^{\mu} P^{\frac{3}{2}} \Lambda_{\mu \nu}^{A, n} P^{\frac{3}{2}} \psi^{v}, \\
\equiv & -\bar{\psi}^{\mu} P^{\frac{3}{2}}\left\{\left(i \not D-m_{\Delta 0}\right) g^{\mu \nu}+i A\left(\gamma^{\mu} D^{v}+\gamma^{v} D^{\mu}\right)\right. \\
& +\frac{i}{n-2}\left[(n-1) A^{2}+2 A+1\right] \gamma_{\mu} \not D \gamma_{v} \\
& +\frac{m_{\Delta 0}}{(n-2)^{2}}\left[n(n-1) A^{2}+4(n-1) A+n\right] \gamma_{\mu} \gamma_{v} \\
& +\frac{g_{\Delta 1}}{2} \psi h \gamma_{5} g_{\mu \nu}+\frac{g_{\Delta 2}}{2}\left(\gamma_{\mu} u_{v}+u_{\mu} \gamma_{v}\right) \gamma_{5} \\
& \left.+\frac{g_{\Delta 3}}{2} \gamma_{\mu} \psi \gamma \gamma_{5} \gamma_{v}\right\} P^{\frac{3}{2}} \psi^{v}, \\
\mathcal{L}_{\pi N \Delta}^{(1)} & =g_{\Delta} \bar{\psi}_{\mu, i} P_{i j}^{\frac{3}{2}}\left(g^{\mu \nu}+\tilde{z} \gamma_{\mu} \gamma v\right) u_{v, j} \Psi+\text { h.c, } \\
& \mathcal{L}_{\Delta}^{(2)}=-c_{1}^{\Delta} \operatorname{Tr}\left(\chi_{+}\right) \bar{\psi}_{\mu, i} P_{i j}^{\frac{3}{2}} g^{\mu \nu} \psi_{v, j} .
\end{aligned}
$$

We can write the bare parameters of the Lagrangians (3), (4) and (5) in renormalized parameters and counterterms as

$$
m_{\Delta 0}=z_{\Delta}+\delta z_{\Delta}, \quad m_{0}=m+\delta m, \quad c_{1,0}^{\Delta}=c_{1}^{\Delta}+\delta c_{1}^{\Delta}, \quad \ldots
$$

where $z_{\Delta}$ is the complex pole of the delta propagator in the chiral limit, and $m$ is the mass of the nucleon in the chiral limit. We use the renormalized mass parameters in the free propagators and improve the counterterms perturbatively. Fig. 1.

The contributions to the delta self-energy up to order $O\left(q^{3}\right)$ from loop diagrams are shown in

To determine the complex pole position $z$ for the delta propagator we solve the equation (where $s$ corresponds to square root of $\mathrm{z}$ in Ref. [9])

$$
z-z_{\Delta}+4 c_{1}^{\Delta} M^{2}-\tilde{\Sigma}_{1}\left(z^{2}\right)-z \Sigma_{6}\left(z^{2}\right)=0,
$$

the pole of delta resonance up to third order is given by

$$
z=z_{\Delta}-4 c_{1}^{\Delta} M^{2}+\left.\left(\Sigma^{(a)}+\Sigma^{(b)}\right)\right|_{p=z_{\Delta}}-\left.\left(\Sigma_{\text {Sub }}^{(a)}+\Sigma_{\text {Sub }}^{(b)}\right)\right|_{p=z_{\Delta}} .
$$



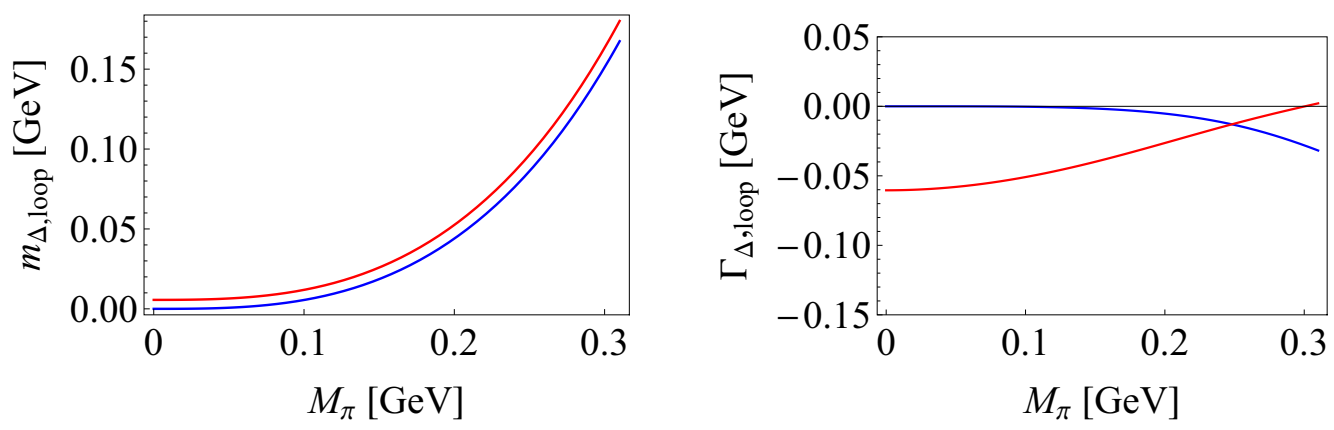

Figure 2. Contributions of the renormalized loop diagrams for real and imaginary part of the pole of delta resonance depending on the pion mass.

We use the numerical values of the parameters as $m_{N}=0.94 \mathrm{GeV}, M_{\pi}=0.14 \mathrm{GeV}, z_{\Delta}=(1.21-$ $\left.\frac{i}{2} 0.10\right) \mathrm{GeV}, F_{\pi}=0.09 \mathrm{GeV}, g_{A}=1.26 \mathrm{GeV}, g_{1}=\frac{9}{5} g_{A} \mathrm{GeV}$ and $g_{\Delta}=1.127 \mathrm{GeV}$. Explicit contributions to the pole of delta resonance are

$$
z=\left[\left(1.210-\frac{i}{2} 0.100\right)-0.078 c_{1}^{\Delta}+\left(0.015-\frac{i}{2} 0.0024\right)\right] \mathrm{GeV} .
$$

We compare the two power counting schemes the CMS and the small-scale expansion (SSE) [6] in Fig. 2. The contributions are represented depending on pion mass to the real and imaginary part of the pole. The red line stands for the results in SSE and the blue line is for the results in CMS. If one uses the SSE the chiral corrections to the real part of the pole are slightly larger in the entire $0<\mathrm{M}_{\pi}<0.3 \mathrm{GeV}$ range.

The authors would like to thank J. Gegelia and D. Djukanovic for valuable contributions to the work. The work of Y. Ü was supported by the Scientific and Technological Research Council of Turkey (TÜBİTAK).

\section{References}

[1] S. Weinberg, Physica A 96, 327 (1979)

[2] J. Gasser and H. Leutwyler, Annals Phys. 158, 142 (1984)

[3] J. Gasser and H. Leutwyler, Nucl. Phys. B 250, 465 (1985)

[4] J. Gasser, M. E. Sainio and A. Svarc, Nucl. Phys. B 307, 779 (1988)

[5] D. Djukanavic et al., Phys. Lett. B 680, 235 (2009)

[6] T. R. Hemmert, B. R. Holstein and J. Kambor, J. Phys. G 24, 1831 (1998)

[7] L. M. Nath, B. Etemadi and J. D. Kimel, Phys. Rev. D 3, 2153 (1971)

[8] W. Rarita and J. S. Schwinger, Phys. Rev. 60, 61 (1941)

[9] C. Hacker et al., Phys. Rev. C 72, 055203 (2005) 